\title{
ACO Technique for Reducing Energy Consumption in Wireless Sensor Network
}

\author{
Madhushree $\mathrm{G}^{1}$, Kavitha $\mathrm{V} \mathrm{N}^{2}$, Arpitha $S \mathrm{M}^{3}$, Latha $S \mathrm{~S}^{4}$, Dr. ArunBiradar ${ }^{5}$ \\ ${ }^{1}$ Student ,EWIT, India, Madhushreeg6@gmail.com \\ ${ }^{5}$ Prof. and HOD of CSE,EWIT, India, arunbiradar@ewit.edu
}

\begin{abstract}
Wireless Sensor Network (WSN) is one of the attractive fields for researchers. There are optimization techniques such as Weighted Compressive Data Aggregation, Cluster-based Weighted Compressive Data Aggregation and Ant Colony Optimization .WCDA and CWCDA are analyzed and discussed. WCDA and CWCDA algorithm is used for reducing energy consumption in WSN model .The simulation results of WCDA and CWCDA algorithms are in terms of dead nodes and packets send to base station nodes. Final simulation results of WCDA and CWCDA shows consumption of energy is minimizes as compared to other approaches.
\end{abstract}

Keywords: WSN, Data Aggregation (DA), Weighted Compressive Data Aggregation (WCDA); Cluster-based Weighted Compressive Data Aggregation (CWCDA); Ant Colony Optimization (ACO).

\section{INTRODUCTION}

A Wireless Sensor Network (WSN) comprises of hundreds or thousands of minimal effort hubs which could either have a settled location or randomly sent to screen nature. each sensor center point require A differentiate detecting, preparing, storing Also correspondence unit.[1] The situation from claiming sensor center points need not be foreordained. This grants irregular sending Previously, distant terrains alternately catastrophe help operations. Because of their constrained power and short range, sensor center points require with collaboratively partake empowers multi-bounce remote correspondence architectures will allow those transmission from claiming their detected What's more gathered information of the nearest base station. Remote networks require aid feeble to security strike because of those show way of the transmission medium. Besides, remote sensor networks acquire an extra lack of protection light centers require aid as often as possible set secured alongside A dangerous alternately unsafe earth the place they would not physically guaranteed.

\subsection{The Characteristics of Sensor Networks}

- Contains a few a huge number of hubs.

- Sensor hubs are inclined to disappointments.

- The topology changes much of the time because of hub disappointments.

- Sensor hubs have compelled assets.

- Sensor hubs for the most part utilize a communicate correspondence worldview.

\subsection{Sensor node structure}

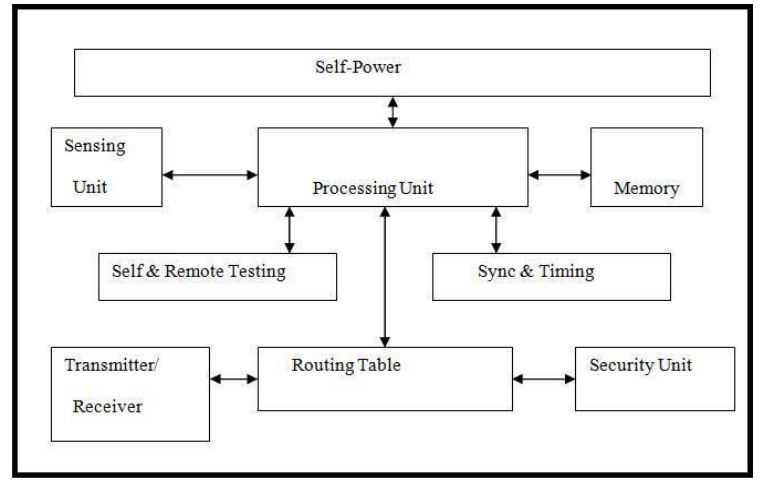

Figure 1 : A Typical Wireless Sensor Node

Figure 1 shows a normal sensor center point. A center point involves about detecting unit, A getting ready unit and memory, A self-control unit Furthermore A remote handset part, and likewise self Also remote testing unit, A synchronizing and timing unit, A coordinating table, and security units. These two units are in charge of figuring the best way to network and security of the information being transmitted .The three main blocks of the sensor node-sensing unit, processing and memory unit, and power unit-

\subsection{Security goals for sensor networks}

In like manner those sensor systems could moreover work Previously, an uncommonly named way the security destinations cover both the people of the standard systems Also targets suiting of the intriguing necessities of exceptionally designated sensor systems. The security objectives are named essential and optional. The essential objectives are referred to 
as standard security objectives, for example, Confidentiality, Integrity, Authentication and Availability (CIAA). The auxiliary objectives are Data Freshness, Self-Organization, Time Synchronization and Secure Localization. The four security objectives for sensor systems are resolved as Confidentiality, Integrity, Authentication and Availability (CIAA).

\subsection{Applications}

The applications for wireless sensor networks are varied, typically involving some kind of monitoring, tracking, or controlling. Specific applications include habitat monitoring, object tracking, nuclear reactor control, fire detection, and traffic monitoring.

\subsection{Energy Optimization in Wireless Sensor Networks}

A standout amongst the real issues in deploying remote sensor system will be with streamline those Vitality utilization same time gathering information starting with the sensor hubs. This is an important class of sensor networks and has many applications such as environmental monitoring, intrusion detection, etc. In such systems, there are four main sources of energy consumption:

- Energy required keeping the communication radios on.

- Energy required for the transmission and reception of control packets.

- Energy required keeping sensors on. And off

- Energy required for actual data transmission and reception.

\section{LITERATURE SURVEY}

In existing work WCDA algorithm is proposed in the mix with CWCDA algorithm for lessening vitality utilization and activity heap of system.

\subsection{WCDA Algorithm}

This algorithm gives control capacity in sensor hubs. This algorithm frames vitality effective directing trees, having a center around stack adjusting issue for enhancing both lifetime and vitality effectiveness. This algorithm consistently chooses gatherer hubs to make the authority tree inside which each gatherer hub totals a metallic component estimations from the relating hopeful hubs and afterward, every authority hub sends the metallic component estimations to the sink hub. WCDA [2] algorithmic control frames vitality practical steering trees with represent considerable authority in the heap adjusting issue to help both day and age furthermore, vitality strength of the system.

\subsection{CWCDA Algorithm}

Existing CS-based information accumulation strategies devours more vitality which are for all intents and purposes not all that doable. Furthermore, the gatherer hubs in WCDA [2] are consistently chosen which can be far from each other. So the given issues are affected to propose another algorithm i.e. CWCDA. This decreases vitality utilization.

CWCDA algorithm chooses competitor hubs related with each selector hubs inside one bunch. The accumulation tress framed with a littler bunch. This causes critical decrease in vitality utilization.

In [11] drawback of Hierarchical routing protocols is data redundancy as all nodes gathers data in a round where an area is sensed by multiple nodes.In [13], solar energy has been considered as energy harvesting strategy.

\section{SYSTEM DESIGN AND ARCHITECTURE}

This section provides a high-level overview of how the functionality and the responsibilities of the system were portioned and then assigned to subgroups or the components or the modules appropriately.

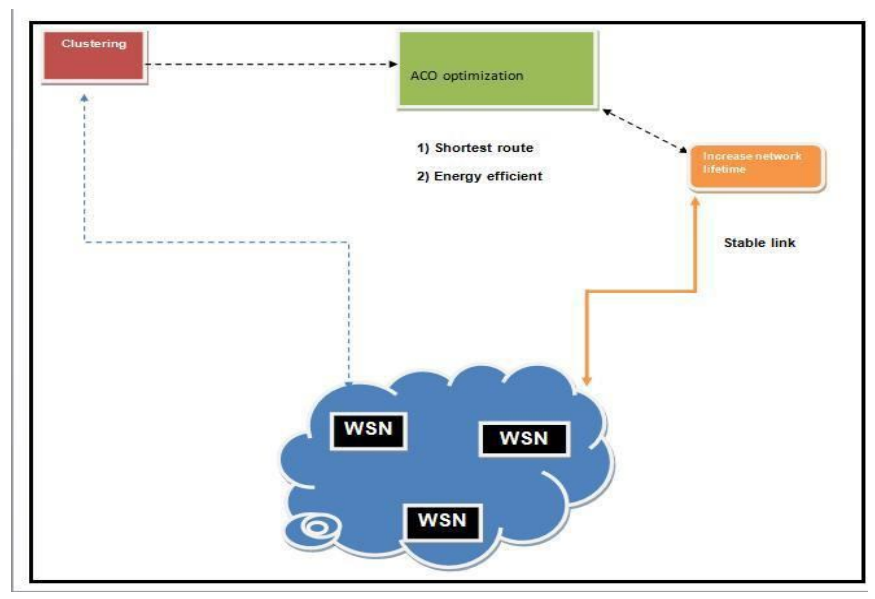

Figure 2 :Architecture Diagram

System design is also recognized as level0 scheme diagrams, describes the general procedure and components which are involved. System design demonstrates how the complete structure is separated as subsystems, all deals through one or many data or controller runs to or from all other, also together provides all functionality of scheme as entire.

Which is used to identify inner data, it must exist in organization to do its job, also demonstrates the flow of data among various portions of organization. Level Zero design is shown in figure. 3.2 give overview of the complete model of the proposed system. 


\section{Level 0: Node Deployment}

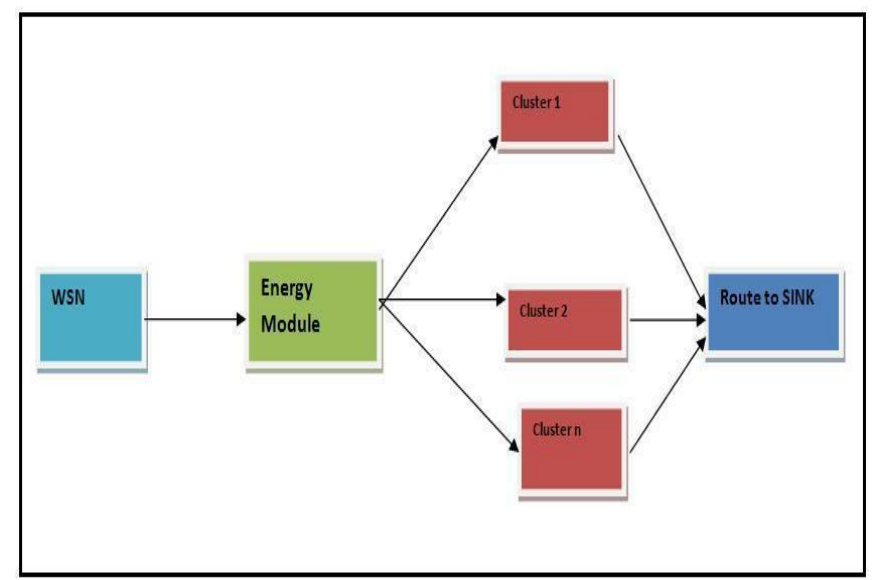

Figure 3 : Node Deployment

Level 1: Cluster Formation

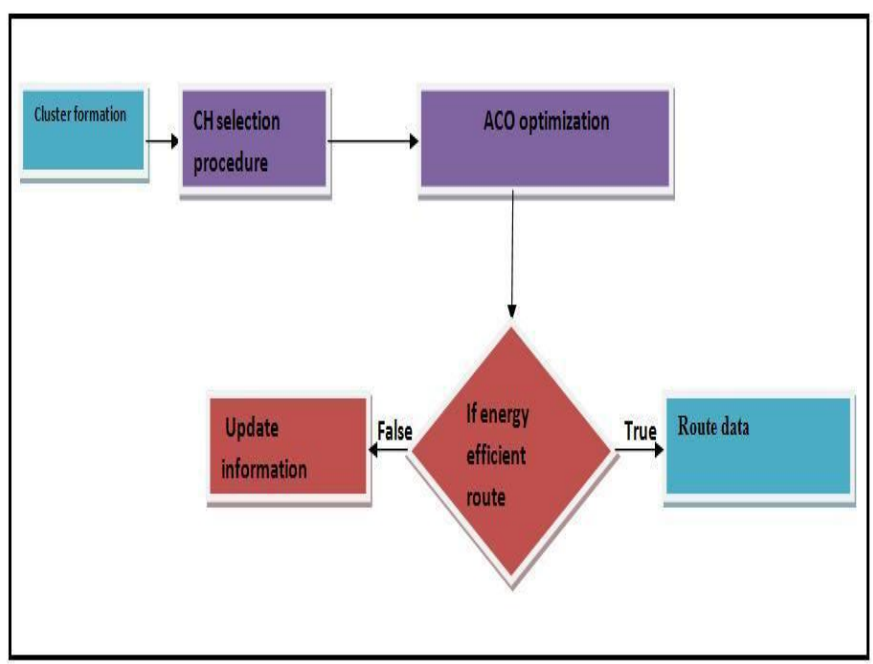

Figure 4 : Cluster Formation

\section{PROPOSED ALGORITHM}

Ant colony change might be a probabilistic procedure for assurance issues which may be acclimated downsize to finding sensible techniques through graph delineation.[3]

\subsection{This algorithm has three phases:}

Route Discovery phase: In this phase new routes are created. The utilization of a Forward (FAnt) and Backward Ant(BAnt) requires a making of new courses. A FAnt is a specialist to the source hub it builds up the pheromone track. a BAnt it builds up the pheromone take to the goal hub. The FAnt is a little bundle and it has a unique arrangement number. Based on grouping number and source address of FAnt hubs can recognize copy bundles. A Forward Ant is communicated by the sender and it is handed-off by neighbors of the sender.
FAnt without precedent for its directing table makes a record.

A record in the directing table is a triple and comprises of pheromone value,destinaion address, and next bounce. The node interprets the source address of Forward Ant as goal address, address of past hub as the following bounce, and contingent upon number of bounces the FAnt expected to achieve the hub and it registers the pheromone esteem. At that point the hub transfers the FAnts to the neighbors. Copied FAnt are recognized by the remarkable succession number and crushed by the hubs. on the off chance that FAnt comes to the destinatin hub, it is prepared extraordinarily.

The goal hub it gathers the data of the FAnt and annihilates it.at a similar time, it makes a BAnt and it sends to the source hub. The BAnt likewise it setting up a track to this node.when the sender gets the BAnt from goal hub to the source hub, the way is set up and information parcels can likewise be sent

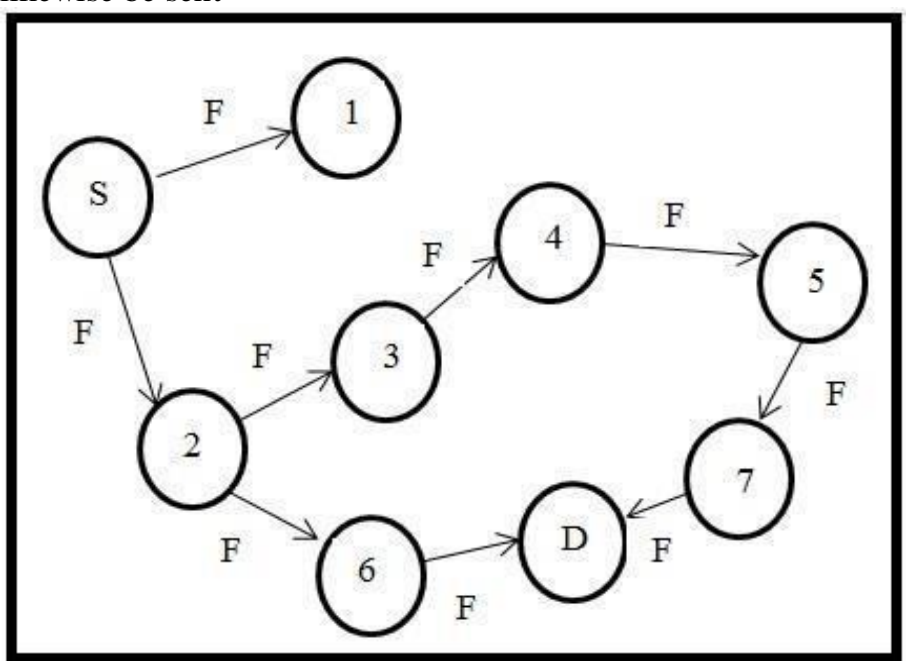

Figure 5 : forward ant

A forward ant $(\mathrm{F})$ is send from source to the target address (destination node) D. Routing table and pheromone values initialize, forward ant which is relayed by other nodes.

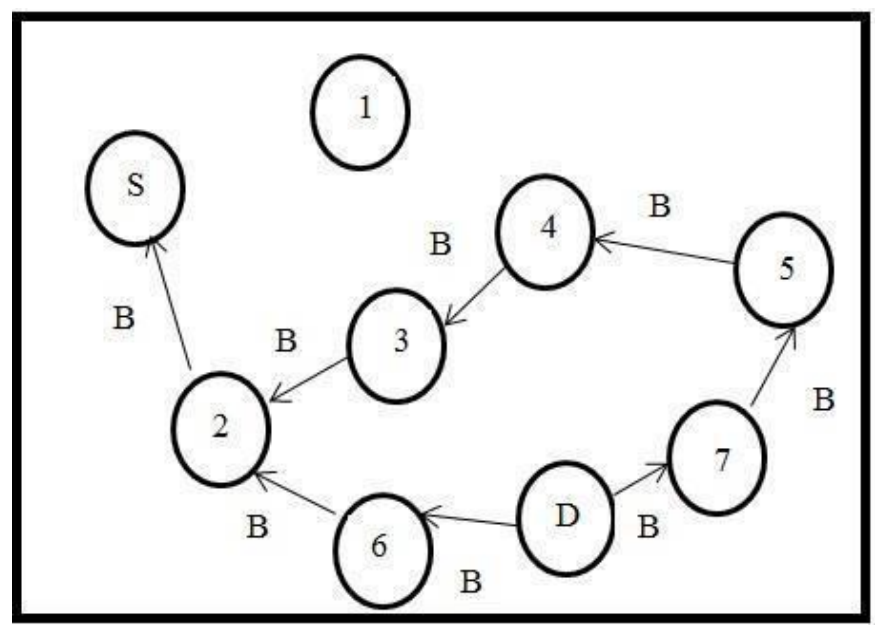

Figure 6 : Backward ant 
The ACO the improvement drawback is transferred into the issues for locating the simplest path on a weighted graph. ACO are applied in numerous improvement drawbacks, array from quadratic course work to macromolecule downfall and routing vehicles and plenty of strategies that are apply for self-motivated issues in real variables, random drawback, multi target and parallel realization. Emmet systemic thought because the initial ACO algorithmic rule, and its aspiration to search out shortest path .The algorithmic rule is comparatively untroubled and it's supported set of ants, every creating one amongst the doable round-trips on the cities .At every stage, the Emmet chooses from one town to a different in step with some rules:

1. It should visit every town specifically once;

2. a foreign town has less likelihood of being chosen (the visibility);

3. The additional intense the secretion path set out on a position between 2 cities, the larger the chance that edge are chosen;

4. Having completed its journey, the hymenopter deposits a lot of pheromones on all edges it traversed,if the journey is short.

\subsection{ACO Meta Heuristic}

Artificial ants utilized in ACO zone unit arbitrary answer development systems that probabilistically manufacture \{answeran answer by iteratively adding arrangement parts to partial arrangements by mulling over (I) heuristic information on the issue case being settled, if offered, and (ii) (artificial) secretion trails that revision dynamically at run-time to reflect the operators' no inheritable hunt ability[5].A lot of vital, the ants' search expertise is accustomed influence in a very method paying homage to reinforcement learning the answer construction in future iterations of the algorithmic program. In addition, the utilization of a colony of ants will provide the algorithmic program multiplied strength and inseveral ACO applications the collective interaction of a population of agents is required to application of ACO algorithms is immense. In theory,[6] ACO is applied to any distinct optimization downside that some answer construction mechanism is planned

. Within the following of this section, we tend to initial outline a generic downside illustration that the ants in ACO exploit to construct solutions, then we tend to detail the ants' behavior whereas constructing solutions, and eventually we tend to outline the ACO Metaheuristic.

\subsection{Ant Behavior}

Ants might be characterized as random construction procedures that build solutions moving on the development graph[7] $\mathrm{G}=(\mathrm{C}, \mathrm{L})$. Ants don't move at random on $\mathrm{G}$, however rather follow a construction policy that might be work of the issue constraints $\Omega$. In general, ants attempt and build possible solutions, however, in the event that necessary, they will generate unworkable solutions. parts $c_{i} \in \mathrm{C}$ and connections $\mathrm{l}_{\mathrm{ij}} \in \mathrm{L}$ will have associated a secretion path $\tau$ ( $\tau \mathrm{i}$ if associated to parts, associated to connections) coding a memory regarding the total discharge search strategy that is refreshed by the ants themselves, and a heuristic price

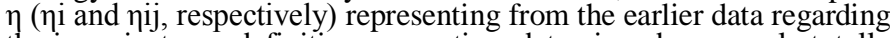
the issue instance definition or run-time data given by a supply totally not quite the same as the ants. In several cases $\eta$ is that the value, or

Associate in nursing appraisal of the value, of extending the present state. These values are utilized by the subterranean insect formula to frame probabilistic selections on an approach to move the graph [9]. More exactly, every emit k of the colony have the subsequent properties:

- It misuses the diagram $\mathrm{G}=(\mathrm{C}, \mathrm{L})$ to search for conceivable arrangements $\mathrm{s}$ of least cost. That is, arrangements $\mathrm{s}$ such $\mathrm{fs}=$ minutes $f(s, t)$.

- It is a memory Mk that it uses to store information with respect to the trail it took after to date. Memory might be utilized

(i) to make conceivable arrangements (i.e., to execute limitations $\Omega$ ),

(ii) to measure the appropriate response found, and

(iii) to backtrack the way in reverse to store emission

- It might be relegated start state $\mathrm{x}_{\mathrm{k}}$ and one or a ton of end condition sk. Normally the starting state is communicated either as a unit length grouping (that is, one component succession), or Associate in Nursing unfilled arrangement. - Once in state $\mathrm{xr}_{\mathrm{r}}=\mathrm{x}_{r}-1$, I, if no end condition is happy, it moves to a hub $\mathrm{j}$ in its neighborhood $\mathrm{N}_{\mathrm{ki}}$, that is, to a state $\mathrm{xr}, \mathrm{j} \in \mathrm{X}$. Regularly, moves towards conceivable states are favored, either by means of appropriately sketched out heuristic esteems

$\eta$, or through the work of the ants' memory.

\subsection{Fitness Function}

If (energy of node high\&\& hop count is minimum) \{ $\Delta \tau=\Delta \tau *$ probability $\}$

By using this formula we calculate fitness of our path and optimize our route.

\subsection{Application}

Ant colony optimization technique has been used in various applications:

- Travelling salesman problem [8]

- Quadratic assignment problem

- Network model problem 
- Vehicle routing problem

\subsection{Advantages}

- Distributed calculation stays away from untimely joining

- The eager heuristic helps see adequate determination inside the early determination inside the beginning times of the inquiry strategy.

-The aggregate association of a populace of specialists.

-Positive Feedback represents quick revelation of fine Solutions.

\section{PERFORMANCE EVALUATION}

Results are simulated on NS2 Simulator.The simulation results are shown in figure below

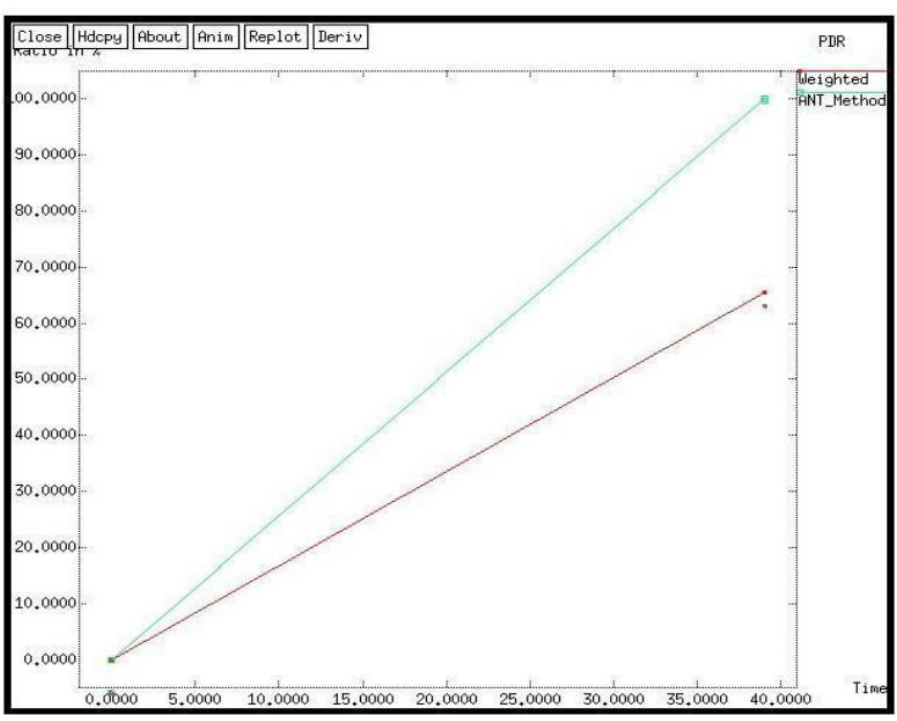

Figure 7 : packet delivery ratio for existing and proposed system

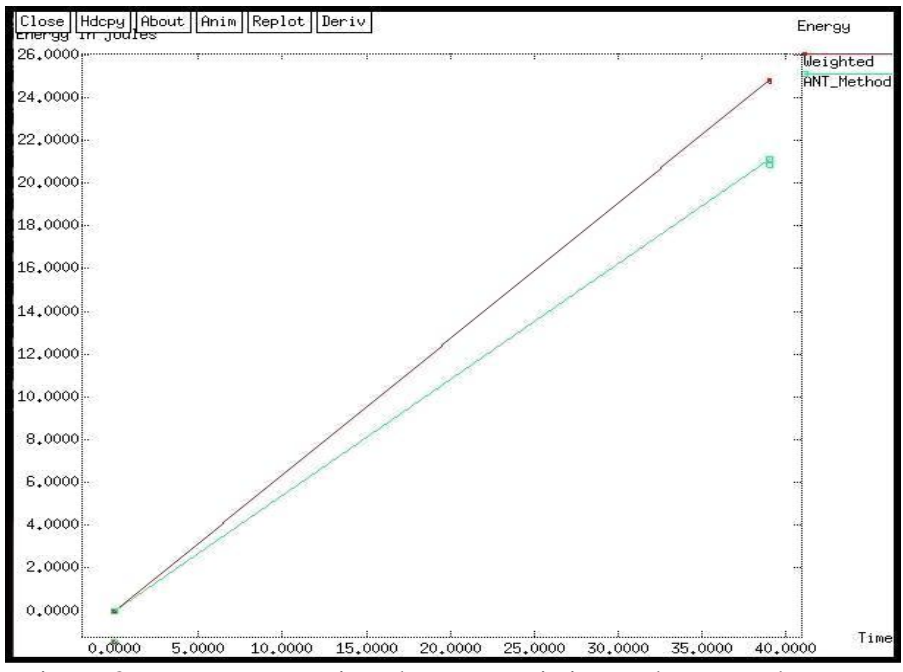

Figure 8 : energy comparison between existing and proposed system

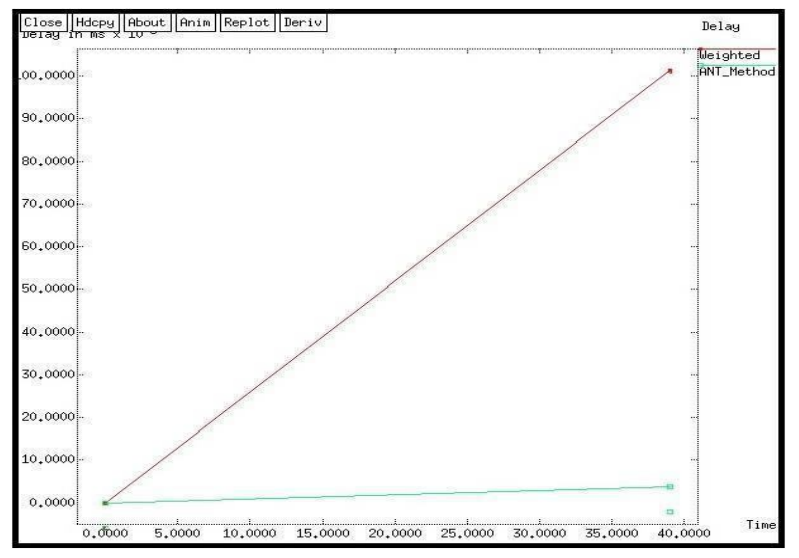

Figure 9: delay comparison

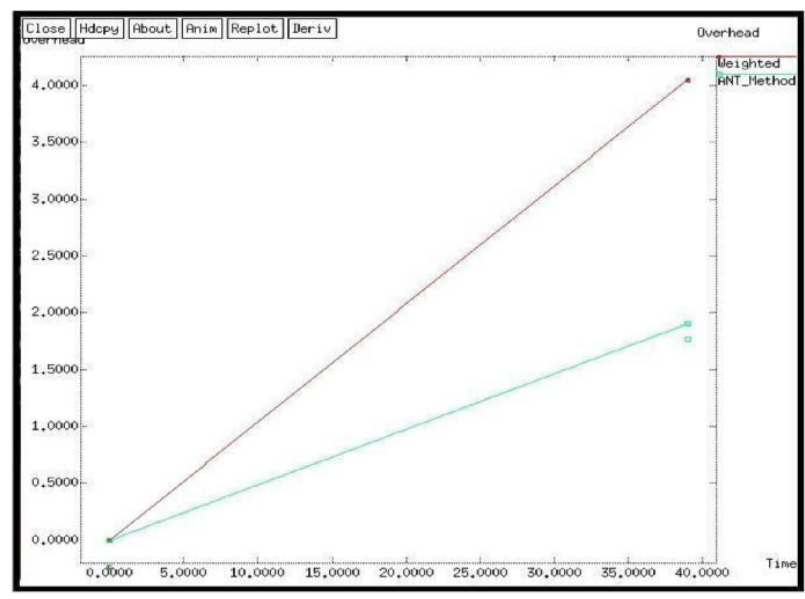

Figure 10 : overhead comparsion

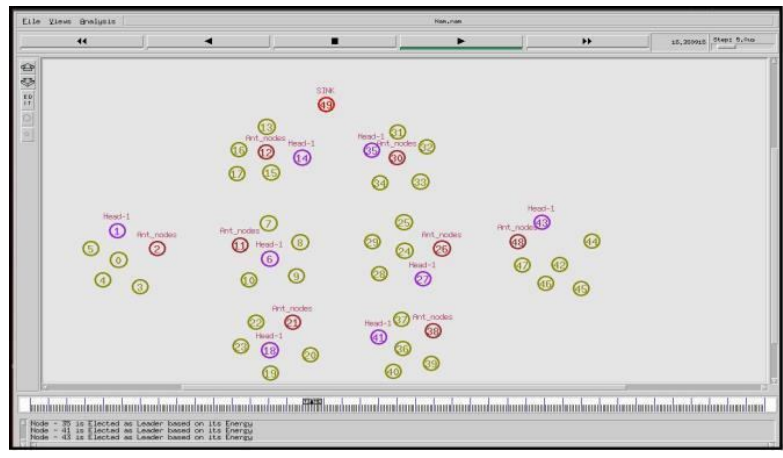

Figure 11 : Simulation topology

\section{CONCLUSION \& FUTURE SCOPE}

In this paper study about sensor network is done and its application result shows that the paper describes the simulation results of previous method CWCDA and their performances. Some of the energy improvements can be done in future work 
Madhushree G et al., International Journal of Computing, Communications and Networking, 7(2) April - June 2018, 42-47

related to deficiencies of previous work. In ACO technique the delay and overhead reduced. In turn packet delivery ratio ,throughput and energy is increased. Hence this increases network lifetime

\section{REFERENCES}

[1]. Sanjeev Kumar Gupta, Poonam Sinha "Overview of Wireless Sensor Network: A Survey" International Journal of Advanced Research in Computer and Communication Engineering Vol. 3,Issue 1, January 2014,pp.5201-5207

[2]. S. Abbasi_Daresari, J. Abouei, "Towards cluster-based weighted compressive data aggregation in wireless sensor networks", Adhoc networks 2015, Vol. 36, part- 1 pp. 368-385.

[3]. Deepthi.N, Bharathi.R "Survey On Ant Colony Optimization (ACO) Algorithm" International Journal of Application or Innovation in Engineering \& Management (IJAIEM) editor@ijaiem.org Volume 4, Issue 9, September 2015,pp.113-117.

[4]. ShabbirHasan , Md. Zair Hussain , R. K. Singh "A Survey of Wireless Sensor Network" International Journal of Emerging Technology and Advanced Engineering, Volume 3, Issue 3, March 2013,pp.487-492.

[5]. Nimisha T.S, R. Ramalakshmi ,", Energy Efficient Connected Dominating set construction using Ant colony optimization Techniques in wireless sensor network". IEEE sponsored 2 International conference on Innovocation in Information embedded and communication system , 978-14799-6818-3/15 (2015) IEEE pp1-5.

[6]. G.Gajalakshmi ,Dr. G. Umaranisrikanth,"a survey on the utilization of ant colony optimization algorithm in WSN."International conference on information communication and embedded system 978-1-5090-2552-7 (ICICES 2016), pp. 1- 4 .

[7]. FareenFarzana A.H, Dr. S.Neduncheliyan,"Ant Based MobilityAided Routing in Mobile WirelessSensorNetworks" International conference On Information Communication And Embedded System 978-1-5090-2552-7(ICICES 2016), pp. 1-5

[8]. Buratti, C., Conti, A., Dardari, D., Verdone, R., An Overview on Wireless Sensor Networks Technology and Evolution. 2009: p., 6869-6896

[9]. KaziChandrima Rahman "A Survey on Sensor Network" JCIT, ISSN 2078-5828 (PRINT), ISSN 2218-5224 (ONLINE),

[10]. VOLUME 01, ISSUE 01, MANUSCRIPT CODE: 100715,pp.76- 87

[11]. Akyildiz, I.F.S., W.Sankarasubramaniam, Y.Cayirci, E., Wireless sensor networks: a survey. Computer Networks, 2002. 38(4): p. 393-422

https://doi.org/10.1016/S1389-1286(01)00302-4
[12] SauravGhosh, SanjoyMondal, UtpalBiswas, "Dominating Set based modified LEACH using Ant Colony Optimization for data gathering in WSN".International Conference on Advances in Electrical, Electronics, Information, Communication and Bio- Informatics 978-1-4673-2 (2016) IEEE.pp390-396

[13 CherifaBoucetta, HanenIdondi, Leila AzouzSaidene,",Ant colony optimization based hierarchical data dissemination in WSN",978-1-4799-5344-8/15(2015) IEEE pp782-787

[14] Avishek Banerjee ,Samiran Chattopadhyay, Anup Kumar Mukhopadhyay, Grigoras Gheorghe "A Fuzzy-ACO

Algorithm to enhance Reliability Optimization through Energy Harvesting in WSN" International Conference on Electrical, Electronics, and Optimization Techniques (ICEEOT) - 2016.7754748 IEEE, pp. 584-589 https://doi.org/10.1109/ICEEOT.2016.7754748

[15] KashifSaleem, Mehmet .A.Orgun, Talal AL-Muhtadi, "Analysis of the Scalibility of an ACO based routing protocol for wireless sensor Network." 2015, 12 International conference onInformation Technology -New Generation. 9781-4799-8828(2015) IEEE, pp. 234-239

\section{AUTHORS BIOGRAPHY}

Dr. Arun Biradar working as professor and HOD of Computer Science in East west institute of Technology affiliated by VTU. His area of Interest are Wireless Ad-hoc network, Computer Networks, Software Engineering, Genetic Algorithm, Machine Learning, IoT, Cloud Computing and Member of Belgaum Board of Examiner(BOE),Visvesvaraya Technological University,Belgaum.

Madhushree G pursuing final year B.E(CSE) in East West Institute of Technology Affiliation by Visvesvaraya Technological University,Belgaum. Area of interest is Computer Networks .

Kavitha $\mathbf{V} \mathbf{N}$ pursuing final year B.E(CSE) in East West Institute of Technology Affiliation by Visvesvaraya Technological University,Belgaum. Area of interest is Computer Networks .

Arpitha S M pursuing final year B.E(CSE) in East West Institute of Technology Affiliation by Visvesvaraya Technological University,Belgaum. Area of interest is Ad-hoc Wireless Networks .

Latha S S pursuing final year B.E(CSE) in East West Institute of Technology Affiliation by Visvesvaraya Technological University,Belgaum. Area of interest is Storage Area Networks 